\title{
EFECTOS DE LAS VARIABLES MACROECONÓMICAS E INSTITUCIONALES EN EL DESEMPLEO: ANÁLISIS PARA EUROPA (1985-2011)*
}

\author{
Recibido: 02 de mayo de 2016 • Aprobado: 26 de noviembre de 2016 \\ DOI: $10.22395 /$ seec.v20n42a1
Ana Cristina Mingorance-Arnáiz ${ }^{* *}$
Antonio Calvo-Bernardino***
Begoña Barruso-Castillo***

\section{RESUMEN}

Este trabajo evalúa la influencia de las variables macroeconómicas e institucionales en la tasa de desempleo de 14 países de la Unión Europea. Se recurre a un modelo de datos de panel para el período 1985-2011. El mismo permite concluir sobre qué variables macroeconómicas e institucionales puede resultar más adecuado actuar para mejorar la respuesta del mercado de trabajo a los problemas económicos. Del análisis se concluye que la protección excesiva a los trabajadores, el salario mínimo interprofesional, la centralización en la negociación salarial y las elevadas cotizaciones empresariales a la Seguridad Social, así como la escasa competencia en el mercado de productos, pueden explicar, en parte, las mayores tasas de desempleo que presentan algunos países de la Unión Europea.

\section{PALABRAS CLAVE}

Mercado laboral; tasa de desempleo; regulación del mercado de trabajo; regulación del mercado de productos; datos de panel; Europa.

\section{CLASIFICACIÓN JEL}

C33; E24; J01; J08

\section{CONTENIDO}

Introducción; 1. Revisión de la literatura existente; 2. Modelo econométrico; 3. Principales resultados; 4. Conclusiones; Bibliografía.

\footnotetext{
Este artículo de reflexión nace con el objetivo de encontrar las razones por las que los países de la Unión Monetaria Europea, pese a aplicar políticas económicas comunes, presentan tasas de desempleo muy diferentes. Conocer los motivos que se esconden tras las elevadas tasas de desempleo que presentan algunos países, así como las medidas que podrían ayudar a reducirlas. Período de ejecución: mayo de 2015-abril de 2016

* Economista, Universidad Complutense de Madrid, Madrid, España. Diplomada en Negocios Europeos, Leeds Metropolitan University, Leeds, Reino Unido. Doctora en Ciencias Económicas, Universidad CEU San Pablo, Madrid, España. Profesora agregada e investigadora, Facultad de Economía, Universidad CEU San Pablo, Madrid, España. Vicedecana, Facultad de Economía, Universidad CEU San Pablo, Madrid, España. Dirección: Cl. Julián Romea \# 23, Madrid 28003, España. Tel.: +34 915140440 Correo electrónico: mingor.fcee@ceu.es.

*** Economista, Universidad Complutense de Madrid, España. Doctor en Ciencias Económicas, Universidad Complutense de Madrid, España. Catedrático e Investigador, Facultad de Economía, Universidad CEU San Pablo, Madrid, España. Rector, Facultad de Economía, Universidad CEU San Pablo, Madrid, España. Dirección: Calle Julián Romea \# 23, Madrid 28003, España. Teléfono: +3491514 0440. Correo electrónico: acalvo@ceu.es.

${ }^{* * * *}$ Economista, Universidad Complutense de Madrid, España. Doctora en Ciencias Económicas, Universidad Complutense de Madrid, España. Profesora agregada e investigadora, Facultad de Economía, Universidad CEU San Pablo, Madrid. Coordinadora académica de la titulación de ADE (Administración y Dirección de Empresas), Facultad de Economía, Universidad CEU San Pablo, Madrid España. Dirección: Cl. Julián Romea \# 23, Madrid 28003, España. Tel: +34 91514 0440. Correo electrónico: barruso.cee@ceu.es.
} 


\section{EFFECTS OF MACROECONOMIC AND INSTITUTIONAL VARIABLES ON UNEMPLOYMENT: THE EUROPEAN CASE (1985-2011)}

\section{ABSTRACT}

This paper evaluates the influence that macroeconomic and institutional variables have on the unemployment rate in 14 countries within the European Union. A panel data model is used for the 1985-2011-time period. The model allows concluding which macroeconomic and institutional variables could be more effective for improving the labor market response when facing economic difficulties. It can be concluded then that excessive protection for workers, inter-professional minimum salary wage, as well as a low competition in the product market, can partially explain the higher unemployment rates found in several countries of the European Union.

\section{KEY WORDS}

Labor market; Unemployment rate; Labor market regulation; product market regulation; panel data; Europe.

\section{JEL CLASSIFICATION}

C33; E24; J01; J08

\section{CONTENT}

Introduction; 1. Existent literature review; 2. Econometric model; 3. Main results; 4. Conclusions; Bibliography.

\section{EFEITOS DAS VARIÁVEIS MACROECONÔMICAS E INSTITUCIONAIS NO DESEMPREGO: ANÁLISE PARA EUROPA (1985-2011)}

\section{RESUMO}

Este trabalho avalia a influência das variáveis macroeconômicas e institucionais na taxa de desemprego de 14 países da União Europeia. Se recorre a um modelo de dados de painel para o período 1985-2011. O mesmo permite concluir sobre que variáveis macroeconômicas e institucionais pode resultar mais adequado atuar para melhorar a resposta do mercado de trabalho aos problemas econômicos. Da análise se conclui que a proteção excessiva aos trabalhadores, o salário mínimo inter-profissional, a centralização na negociação salarial e as elevadas cotizações empresariais à Segurança Social, assim como a escassa competência no mercado de produtos, podem explicar em parte as maiores taxas de desemprego que apresentam alguns países da União Europeia.

\section{PALAVRAS CHAVE}

Mercado laboral; Taxa de desemprego; regulação do mercado de trabalho; regulação do mercado de produtos; Dados de painel; Europa.

\section{CLASSIFICAÇÃO JEL}

C33; E24; J01; J08

\section{CONTEÚDO}

Introdução; 1. Revisão da literatura existente; 2. Modelo econométrico; 3. Principais resultados; 4. Conclusões; Bibliografia. 


\section{INTRODUCCIÓN}

La crisis económica iniciada en el verano del 2007 ha tenido efectos negativos en el mercado de trabajo de casi todos los países de la Unión Europea (UE). Pero mientras en unos el desempleo se ha disparado (España, Grecia, Irlanda y Portugal), en otros la situación previa a la crisis apenas se ha modificado (Finlandia y Bélgica). Llaman la atención los casos de Austria y Alemania porque, pese a la crisis, han logrado reducir su tasa de desempleo.

Este comportamiento tan diferente del mercado de trabajo ante un mismo shock lleva a plantearse algunas cuestiones a las que se tratará de dar respuesta a lo largo del presente trabajo, entre otras, ¿qué parte del crecimiento del desempleo es coyuntural y cuál estructural?, ¿qué hace que el mercado de trabajo de unos países se vea más afectado por los shock que el de otros?, ¿qué debilidades económicas presentan los países con tasas de desempleo más elevadas?, ¿qué deberían hacer esos países para corregir su desequilibrio laboral?

Para dar respuesta a estas cuestiones, y tras consultar la literatura existente al respecto, se ha construido un panel de datos para el período 1985-2011 constituido por las principales variables, tanto macroeconómicas como institucionales, que se consideran causantes del desempleo, o que, al menos, influyen en su persistencia a lo largo del tiempo, en los 14 países seleccionados. Del análisis se desprende que mientras las variables macroeconómicas, como la caída del crecimiento económico, el comportamiento de la productividad de la mano de obra, y una estructura productiva basada en sectores intensivos en mano de obra de baja cualificación, resultan esenciales para generar desempleo, son las variables institucionales, y más en concreto la excesiva regulación del mercado de trabajo y de productos, la existencia de un salario mínimo interprofesional elevado y las altas cotizaciones empresariales a la seguridad social las que explican la persistencia del desempleo a lo largo del tiempo.

El trabajo se ha dividido en cuatro partes. Tras esta introducción, se recoge, en la primera parte, la evolución del desempleo en los países objeto de estudio, así como un resumen de la literatura que analiza los problemas del mercado de trabajo, sus causas y las posibles medidas para su solución, haciéndose referencia, en su mayor parte, a un trabajo previo de los autores. En la segunda se describe la base de datos que se ha construido, así como la metodología seguida en el estudio. En la tercera sección se presentan los resultados empíricos obtenidos y los test de robustez realizados. Finalmente, el trabajo se cierra con un último apartado de conclusiones y propuestas. 


\section{REVISIÓN DE LA LITERATURA EXISTENTE}

Una rápida revisión de la información presentada en la tabla 1, donde se recoge el comportamiento de la tasa de desempleo para un período de tiempo superior a los 50 años en 14 países de la UE, permite extraer las primeras conclusiones sobre la temporalidad o permanencia del desempleo a lo largo del tiempo. Así, se puede afirmar, por un lado, que la tasa de desempleo es una de las variables macroeconómicas más afectadas por los ciclos económicos, y por otro, que es también una de las que más varía entre países.

Si bien, antes de buscar las causas que pueden justificar este comportamiento tan distinto entre países, es necesario ofrecer una visión, aunque sea somera, de las teorías hasta ahora desarrolladas, que tratan de encontrar las causas del desempleo y los motivos que hacen que en unos países la tasa de desempleo sea mayor que en otros. Estas teorías que nacieron en la década de 1970 han evolucionado con el tiempo, y si bien en un principio buscaron en la insuficiencia de la demanda agregada la justificación al desempleo, en la década de 1990 encontraron en los factores institucionales del mercado de trabajo un factor adicional de explicación de los niveles de desempleo.

Tabla 1. Tasa media de desempleo por períodos (Datos en porcentajes)

\begin{tabular}{|l|c|c|c|c|c|c|}
\hline \multicolumn{1}{|c|}{ País } & $1960-1975$ & $1976-1984$ & $1985-1996$ & $1997-2007$ & $2008-2012$ & $1960-2015$ \\
\hline Alemania & 0,99 & 4,73 & 5,98 & 8,8 & 6,09 & 4,92 \\
\hline Austria & 1,42 & 2,28 & 3,62 & 4,29 & 5,13 & 3,12 \\
\hline Bélgica & 2,56 & 9,59 & 9,17 & 7,8 & 7,97 & 6,91 \\
\hline Dinamarca & 1,98 & 6,52 & 7,55 & 4,69 & 6,6 & 5,09 \\
\hline España & 2,62 & 11,49 & 20,11 & 12,69 & 21,08 & 12,41 \\
\hline Finlandia & 2,01 & 5,33 & 9,08 & 9,39 & 8,19 & 6,39 \\
\hline Francia & 2,19 & 6,68 & 10,62 & 10,22 & 9,5 & 7,27 \\
\hline Grecia & 4,18 & 3,79 & 7,63 & 10,22 & 14,36 & 7,11 \\
\hline Irlanda & 5,36 & 10,13 & 15,2 & 5,52 & 12,35 & 9,26 \\
\hline Italia & 3,31 & 4,78 & 8,45 & 9,05 & 8,41 & 6,51 \\
\hline Países Bajos & 1,83 & 7,91 & 7,81 & 3,72 & 5,19 & 4,94 \\
\hline Portugal & 2,02 & 6,96 & 6,28 & 6,01 & 12,94 & 7,72 \\
\hline Suecia & 1,49 & 2,01 & 4,76 & 6,79 & 7,57 & 4,13 \\
\hline Reino Unido & 3,02 & 8,27 & 9,25 & 5,41 & 7,33 & 6,24 \\
\hline
\end{tabular}

Fuente: Layard, Nickell y Jackman (1991) y OECD 
Son estos factores institucionales los que hoy día gozan de mayor intensidad a la hora de explicar la permanencia del desempleo. Así, algunos trabajos recientes que pueden añadirse a los mencionados en Pampillón y Mingorance (2014) señalan la duración del seguro de desempleo como factor explicativo de las altas tasas de desempleo (véase Barbanchon (2012) y Farber y Valletta (2013)), mientras que Blanchard, Joumotte y Loungani (2013) afirman que es el llamado taxwedge, diferencia entre el costo del trabajador para el empresario y el salario neto realmente percibido por el trabajador, el que incrementa los costos laborales para la empresa y reduce el empleo. Por su parte, Pérez y Yao (2012) exponen que las reglamentaciones del mercado de trabajo y del mercado de bienes y servicios influyen de manera significativa en el nivel de desempleo existente en las economías.

Como se observa, los trabajos sobre las causas del desempleo son múltiples, como también lo son los estudios que analizan las opciones de flexibilizar el mercado de trabajo para reducir el desempleo. En este sentido el reciente trabajo de Adascalitei y Pignatti (2015) señala que la probabilidad de que se adopten reformas que flexibilicen el mercado de trabajo depende, con carácter general, del nivel de desempleo, del PIB per cápita y del crecimiento del PIB, si bien, deben establecerse diferencias entre las distintas medidas liberalizadoras; así, por ejemplo, para flexibilizar el sistema de negociación salarial es fundamental la deuda pública del gobierno, al igual que ocurre a la hora de reducir la contratación temporal, mientras que en los despidos colectivos, la tasa de desempleo es la variable esencial.

Cacciatore et al., (2016) toma partido en el debate que ha surgido entre desregular el mercado de trabajo o el de productos para reducir el desempleo al afirmar que, aunque a corto plazo la desregulación del mercado de productos puede afectar negativamente al mercado de trabajo, lo cierto es que a largo plazo los efectos son beneficiosos e incluso más intensos que los que surgen con la desregulación del mercado de trabajo. En este sentido, y con carácter general, para reducir el desempleo es preferible actuar sobre el mercado de productos que sobre el mercado de trabajo.

\section{Principales hechos estilizados}

Como se ha podido comprobar, a lo largo del tiempo ha cambiado ligeramente la opinión de los economistas sobre las variables causantes del desempleo. Mientras las variables macroeconómicas gozaron de gran relevancia en las décadas de 1960 y 1970, los nuevos avances de la teoría han hecho que estas variables, que seguían y siguen explicando el desempleo, pasasen a un segundo plano, cobrando mayor fuerza las variables institucionales. 
A continuación, y antes de adentrarnos en el análisis econométrico se muestra, de manera resumida, el comportamiento de las principales variables consideradas en el análisis. Así, en la tabla 2, para el período 1985-2011, se presentan los valores medios que las mismas alcanzan en cada uno de los países considerados, así como los principales estadísticos de las mismas. Los valores muestran la existencia de disparidades importantes entre países y a lo largo del tiempo'.

En primer lugar, en lo que a las variables macroeconómicas se refiere, las discrepancias son marcadas. Respecto a la tasa de inflación cabe señalar las mayores tasas medias de Grecia, España y Portugal, así como los menores niveles de Alemania y Países Bajos. Además, se observa una desaceleración general a lo largo del tiempo que contrasta con la estabilidad de Austria, Alemania, Países Bajos y Reino Unido. El valor máximo se alcanza en Grecia en 1986, y el mínimo en Irlanda, durante la última crisis económica, cuando varios países han alcanzado tasas de inflación negativas.

Respecto al crecimiento económico, Irlanda, seguida a bastante distancia de España, presenta las tasas medias más elevadas para el período considerado, mientras las más bajas corresponden a Italia, Dinamarca y Grecia. Por su parte, la estabilidad en el crecimiento corresponde a Bélgica y Austria, que destacan frente a Finlandia, Grecia e Irlanda, que presentan fluctuaciones más marcadas. De hecho los valores máximos y mínimos los alcanzan Irlanda a medidos de la década de 1990, y Finlandia, durante la crisis de 2007, respectivamente.

En cuanto a la productividad de la mano de obra los mayores crecimientos de la misma a lo largo del período se producen en Finlandia, Suecia y Reino Unido, países que superan el 1,2\% de crecimiento medio anual, mientras, los crecimientos medios más bajos corresponden a Países Bajos (0,24 \%), Alemania (0,30 \%) y Bélgica $(0,46$ \%). Sólo España ha logrado, durante todo el período de crisis, alcanzar tasas de crecimiento de la productividad positivas que descansan en la destrucción de empleo poco cualificado, mientras Grecia muestra tasas de crecimiento de la productividad negativas durante una parte importante del período (un tercio de los años considerados). No obstante, es Alemania quien presenta la tasa de crecimiento más baja $(-12,77 \%)$ en el año 1991.

En relación con la imposición, los mayores crecimientos medios en los impuestos indirectos se han producido en Italia, Países Bajos, España y Suecia, mientras que

\footnotetext{
1 Este hecho no debe sorprender. La variables causantes del desempleo lo son en todos los países con mayor o menor intensidad, si bien, el hecho de que algunos países hayan hecho bien sus deberes y presenten valores adecuados en las variables que generan desempleo, hace que presenten en general tasas de desempleo más moderadas que las de aquellos países que no han hecho adecuadamente sus deberes.
} 
Austria y Reino Unido los han visto caer. Pese a ello, el crecimiento de los impuestos indirectos en la UE entre 1985 y 2011 (1,08 \%) supera con creces al que han experimentado los principales países de la OECD que no son europeos $(0,43 \%)$. No obstante, y dentro de los primeros, son los países de la cuenca mediterránea (Grecia, España, Italia, Portugal, Francia e Irlanda), los que más han incrementado su carga impositiva (1,42 \%).

Por su parte, las variables institucionales describen, en general, comportamientos similares a los de las variables macroeconómicas. El grado medio de centralización en la negociación salarial de los países de la cuenca mediterránea se aproxima, para el período 1985-2011, a valores medios (entre 2,5 y 3,5). La única excepción es Francia, cuyo grado de centralización se sitúa en un nivel medio bajo (2). Mientras, el resto de países de la UE alcanza valores medios más extremos, de clara centralización, caso de Finlandia, Alemania, Suecia y Países Bajos, o descentralización total, en el caso de Reino Unido.

Algo similar ocurre con la legislación que los gobiernos han desarrollado para proteger a los trabajadores. Así, la legislación es más protectora en Alemania, Suecia, España, Italia, Grecia y Portugal, que en el resto de países de la UE. Si bien, son Irlanda y Reino Unido, países de corte anglosajón, los que muestran legislaciones más flexibles.

Respecto al salario mínimo, destaca la inexistencia del mismo durante el período considerado, en Austria, Dinamarca, Alemania, Italia, Finlandia y Suecia. Por su parte, y en relación con el gasto en políticas activas, destacan las continuas fluctuaciones que esta variable experimenta a lo largo del período considerado, así como que España es el país que, en media, mantiene un menor nivel de gasto, y ello pese al fuerte crecimiento global mostrado a lo largo del período, equivalente a los de Dinamarca y Reino Unido.

En cuanto a la densidad sindical, indica que las disparidades son enormes. Destacan los países nórdicos por su mayor nivel de sindicalización frente al bajo nivel de afiliación en Francia y España. Además, en este último país es en el único en el que el nivel de sindicalización crece a lo largo del período 1985-2011. En relación con las cotizaciones empresariales a la Seguridad Social, es importante destacar el caso de Dinamarca por la inexistencia de las mismas. Por el contrario, los niveles más altos se dan en Francia, Suecia, Italia, Finlandia y Bélgica.

En relación con las variables institucionales del mercado de productos, es importante señalar que, con independencia del indicador utilizado, los países europeos presentan mayores barreras a la creación de empresas, y al funcionamiento de la libre competencia que otros países OECD. Destacan los casos de Francia, Grecia, Italia, 
Irlanda, y Portugal por su regulación general y sus elevadas barreras empresariales. No obstante, el esfuerzo realizado en los últimos años, especialmente a comienzos del siglo XXI, ha permitido que estos países alcancen niveles de regulación más próximos a los de los países de su entorno.

Finalmente, y en lo que a la formación de la mano de obra se refiere, resulta valioso indicar que las diferencias entre países son altas, y mientras en algunos países los años medios de formación para el período total superan los 13 años, caso de Alemania, en otros, como en Portugal, no se alcanzan los 7. Sí puede, sin embargo, afirmarse que, con carácter general, son España, Grecia, Italia, Irlanda y Portugal, los países en los que sus ciudadanos dedican menos años a su educación.

Tabla 2. Valores medios y estadísticos descriptivos básicos de las variables consideradas en el estudio (1985-2011)

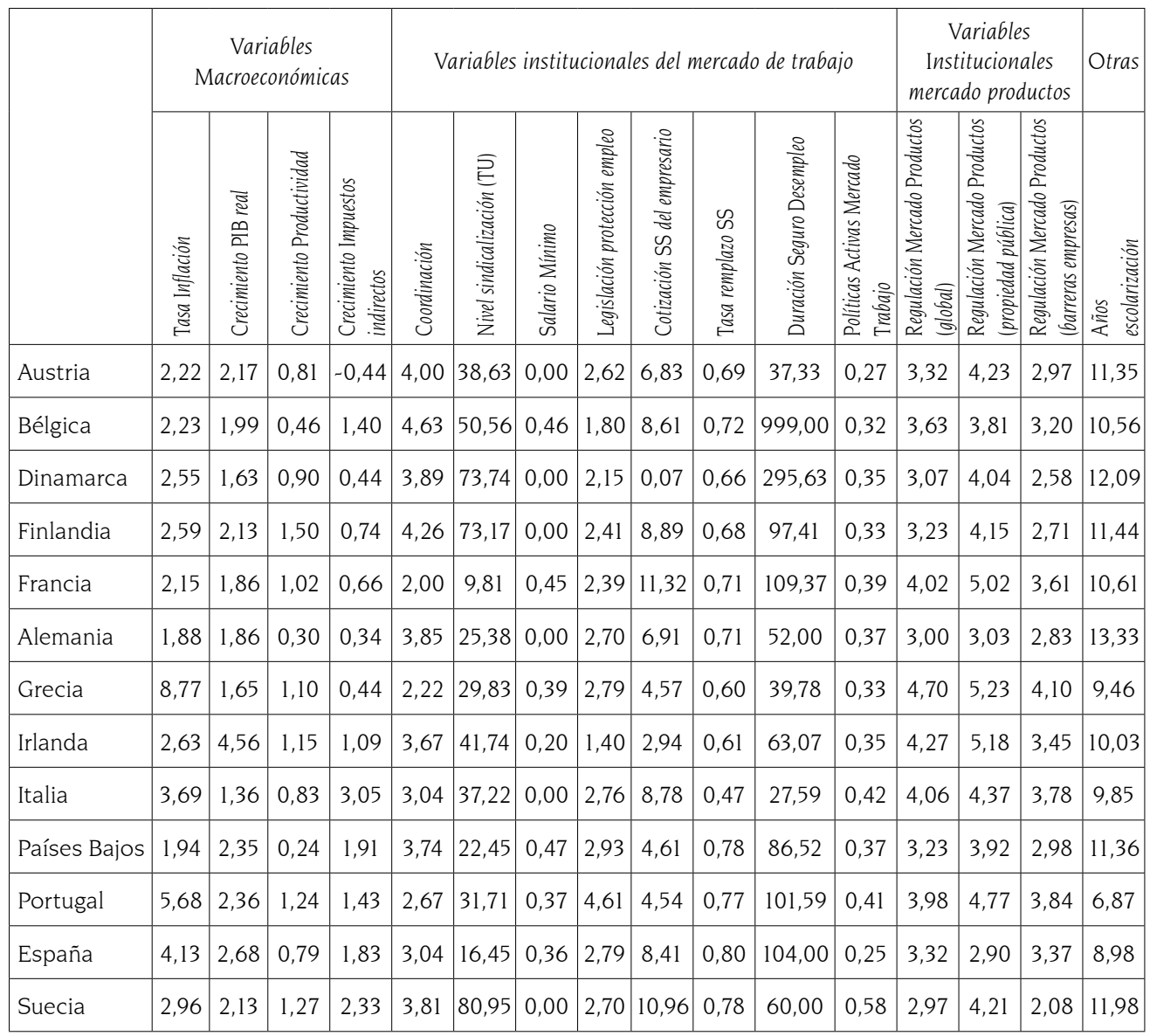


Efectos de las variables macroeconómicas e institucionales en el desempleo: análisis para Europa (1985-2011)

\begin{tabular}{|c|c|c|c|c|c|c|c|c|c|c|c|c|c|c|c|c|}
\hline & \multicolumn{4}{|c|}{$\begin{array}{c}\text { Variables } \\
\text { Macroeconómicas }\end{array}$} & \multicolumn{8}{|c|}{ Variables institucionales del mercado de trabajo } & \multicolumn{3}{|c|}{$\begin{array}{c}\text { Variables } \\
\text { Institucionales } \\
\text { mercado productos }\end{array}$} & \multirow[b]{2}{*}{$\begin{array}{l}\text { Otras } \\
\end{array}$} \\
\hline & 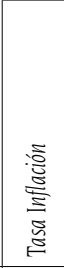 & 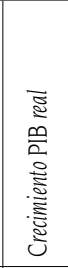 & 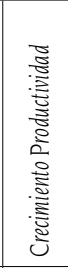 & 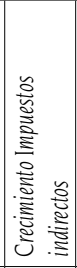 & : & 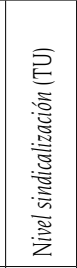 & 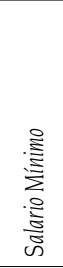 & 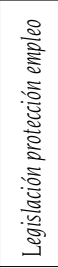 & 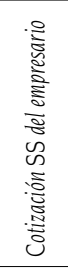 & 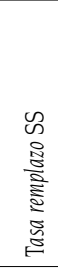 & 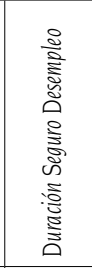 & 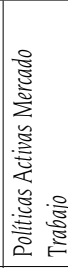 & 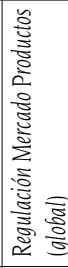 & 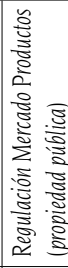 & 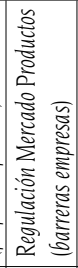 & \\
\hline Reino Unido & 3,69 & 2,24 & 1,21 & $-0,16$ & 1,00 & 36,17 & 0,17 & 1,11 & 3,51 & 0,40 & 36,59 & 0,45 & 1,93 & 1,63 & 1,83 & 12,86 \\
\hline Media & 3,36 & 2,21 & 0,92 & 1,08 & 3,27 & 40,56 & 0,21 & 2,51 & 6,50 & 0,67 & 150,71 & 0,37 & 3,48 & 4,03 & 3,10 & 10,77 \\
\hline Máximo & 23,12 & 10,75 & 4,89 & 30,95 & 5,00 & 91,12 & 0,59 & 5,00 & 14,01 & 0,95 & 999,00 & 0,72 & 6,00 & 6,00 & 6,00 & 13,69 \\
\hline Mínimo & $-1,72$ & $-9,35$ & $-12,77$ & $-18,44$ & 1,00 & 8,47 & 0,00 & 1,03 & 0,00 & 0,15 & 22,00 & 0,10 & 0,93 & 0,83 & 0,30 & 5,40 \\
\hline $\begin{array}{l}\text { Desviación } \\
\text { Estándar }\end{array}$ & 3,19 & 2,49 & 1,51 & 5,59 & 1,13 & 21,72 & 0,21 & 0,82 & 3,20 & 0,13 & 246,60 & 0,11 & 1,50 & 1,31 & 1,82 & 1,76 \\
\hline
\end{tabular}

Fuente: elaboración propia con datos de IMF, OECD, Comisión Europea, Nickell (2006), Visser (2013), Scruggs, Jahn y Kuitto (2013), Conway y Nicoletti (2006), Cohen y Soto (2007) y Cohen y Leker (2014).

\section{MODELO ECONOMÉTRICO}

Para el análisis econométrico, se define el modelo empírico del desempleo a través de un panel de 14 países de la OECD, en concreto países miembros de la Unión Europea, construido con datos anuales de las variables para el período 1985-2011². Los datos de panel, al combinar una dimensión temporal con otra transversal, permiten capturar la heterogeneidad no observable que existe entre los países, al tiempo que permiten estudiar de mejor manera la dinámica del proceso de ajuste . $^{2}$

2 En concreto se incluyen los siguientes países: Austria, Bélgica, Dinamarca, Finlandia, Francia, Alemania, Grecia, Irlanda, Italia, Países Bajos, Portugal, España, Suecia y Reino Unido, todos ellos países de la Unión Europea que presentan algunas características comunes, pero que sobre todo aplican políticas comunes o muy similares. Estas medidas resultan imprescindibles para evitar shocks asimétricos que podrían en duda la estabilidad de la Unión, así como para lograr una convergencia nominal y real que aparece recogida en el Tratado constitutivo de la Unión. Los autores suministrarán la base de datos construida y utilizada en el estudio a cualquier investigador interesado, mediante solicitud al correo electrónico: mingor.fcee@ceu.es.

${ }^{3}$ El hecho de que los países analizados formen parte de una Unión Económica en la que ciertas políticas están hipotecadas (no pueden utilizarse con total libertad por parte de los países) y en la que al mismo tiempo sea necesaria una cierta convergencia nominal y real para evitar shocks asimétricos que en caso contrario generarían inestabilidades en el conjunto de países, hace que conocer un patrón de actuación común para todos los países que permita actuar sobre la tasa de desempleo, sea de utilidad. En cualquier caso debemos ser conscientes de que este patrón o conjunto de variables sobre las que actuar no ayudaría a eliminar el desempleo en todos los países. Por este motivo el modelo arroja los 
El crecimiento del desempleo queda explicado por su propio retardo, de modo que la variación del desempleo depende, entre otras variables, de su evolución. Se admite que existe cierta persistencia en el crecimiento del desempleo. Se considera, además, una serie de variables macroeconómicas e institucionales que ya han sido expuestas por otros autores. Así, la ecuación utilizada para estimar el crecimiento del desempleo es:

$$
\mathrm{Y}_{i t}=\beta \mathrm{Y}_{i t-1}+\gamma \mathrm{X}_{i t}+\mu_{i}+\varepsilon_{i t}
$$

Siendo $Y_{i t}$ la variable dependiente en el país i en el año t. Por su parte $X_{i t}$ hace referencia al vector de variables independientes (macroeconómicas e institucionales del mercado de trabajo y de productos) en cada uno de los países y para cada período de tiempo ${ }^{4}$. También se incluyen en el modelo, efectos fijos $\left(\mu_{i}\right)$ que recogen la heterogeneidad que presentan los países, así como un término error $\left(\varepsilon_{i t}\right)^{5}$.

El período elegido es el comprendido entre 1985 y 2011. Se ha optado por iniciar el estudio en 1985 porque es el momento en que España y Portugal firman su tratado de adhesión a la Unión Europea, incorporándose un año más tarde en 1986. De este modo, todos los países considerados en el estudio, son miembros de la Unión Europea desde el inicio del período de estudio. Se finaliza en 2011 por ser el último año del que se disponen de datos para algunas variables, en concreto para las de carácter institucional.

El concepto de todas las variables consideradas, tanto macroeconómicas como institucionales, así como lo que representan las mismas y el resultado que se espera que tengan en el desempleo, se recoge en las tablas 3 y 4 . En estas mismas tablas se resume el modo en que se ha construido cada serie y se describe cuáles fueron las fuentes de origen de la información del período 1985-2011.

denominados efectos individuales que nos indica que existen variables adicionales, distintas a las consideradas en el modelo, sobre las que habría que actuar para lograr una reducción más intensa del desempleo en aquellos países en los que es necesario.

4 Se debe ser conscientes de que esta forma de proceder nos permite extraer conclusiones generales para el conjunto de países considerados. Las peculiaridades que presenta cada país pueden hacer que su comportamiento, en un momento concreto, pueda diferir del comportamiento medio, que en cualquier caso es válido en el largo plazo y con carácter general. Como consecuencia de ello, las medidas que se propongan, aunque exitosas en su conjunto, pueden no serlo en un momento concreto para algunos de los países considerados.

${ }^{5}$ Los efectos individuales, en este caso considerados fijos, nos permiten conocer el comportamiento particular de cada uno de los países que forman parte de la muestra, y por lo tanto, qué países necesitan medidas adicionales y/o más intensas para alcanzar tasas de desempleo similares a las de aquellos que tienen los niveles más bajos. 
Efectos de las variables macroeconómicas e institucionales en el desempleo: análisis para Europa (1985-2011)

Tabla 3. Variables macroeconómicas consideradas en el estudio

\begin{tabular}{|c|c|c|c|}
\hline Variable & Concepto & $\begin{array}{c}\text { Efecto esperado en la tasa de } \\
\text { desempleo }\end{array}$ & Serie \\
\hline $\begin{array}{l}d u_{i t} \\
d u_{i t}=\operatorname{Ln}(u)_{t}-\operatorname{Ln}(u)_{t-1}\end{array}$ & $\begin{array}{l}\text { Variación de la tasa de des- } \\
\text { empleo media, medida en } \\
\text { términos decimales. Se ha } \\
\text { calculado a partir de la tasa } \\
\text { de desempleo. }\end{array}$ & & (1) \\
\hline $\inf _{i t}=\frac{\mathrm{IPC}_{t}-\mathrm{IPC}_{t-1}}{\mathrm{IPC}_{t-1}}$ & $\begin{array}{l}\text { Tasa de inflación anual cal- } \\
\text { culada a partir del índice de } \\
\text { precios al consumidor. }\end{array}$ & $\begin{array}{l}\text { Es de esperar una relación ne- } \\
\text { gativa entre la inflación y la tasa } \\
\text { de desempleo, lo que pondría } \\
\text { de manifiesto el cumplimiento } \\
\text { de la curva de Phillips. Si el } \\
\text { signo no fuese negativo podría } \\
\text { deberse a la existencia de una } \\
\text { cierta estanflación. }\end{array}$ & (2) \\
\hline $\operatorname{prod}_{i t}$ & $\begin{array}{l}\text { Tasa de crecimiento de la } \\
\text { productividad de la mano } \\
\text { de obra, obtenida como } \\
\text { el porcentaje de cambio } \\
\text { del PIB real por persona } \\
\text { empleada, multiplicada por } \\
1 \text { menos el grado medio de } \\
\text { apertura de la economía. }\end{array}$ & $\begin{array}{l}\text { Es de esperar que un creci- } \\
\text { miento de la productividad } \\
\text { haga menos necesario contra- } \\
\text { tar a trabajadores, por lo que } \\
\text { la tasa de desempleo debería } \\
\text { aumentar. Además, al multi- } \\
\text { plicar la productividad por (1 } \\
\text { menos el grado de apertura de } \\
\text { la economía) estamos supo- } \\
\text { niendo que el crecimiento de la } \\
\text { productividad es menos eficaz } \\
\text { para solventar problemas de } \\
\text { desempleo en una economía } \\
\text { cerrada que en una abierta. }\end{array}$ & (3) \\
\hline $\begin{array}{l}\mathrm{PIB} g_{i t} \\
\mathrm{PIB} g_{i t}=\mathrm{L} n(\mathrm{PIB})_{t}-\mathrm{L} n(\mathrm{PIB})_{t-1}\end{array}$ & $\begin{array}{l}\text { Tasa de crecimiento del } \\
\text { PIB real, calculada como la } \\
\text { diferencia de los logaritmos. }\end{array}$ & $\begin{array}{l}\text { Es de esperar que las econo- } \\
\text { mías con mayor crecimiento } \\
\text { muestren niveles de desempleo } \\
\text { más bajos, por lo que el signo } \\
\text { debería ser negativo. }\end{array}$ & (4) \\
\hline $\begin{array}{l}\text { itax }_{\text {it }} \\
\text { itax }_{\text {it }}=\frac{\text { imp.indirectos }_{t}-\text { subsidios }_{t}}{\text { consumodelas familias. }}\end{array}$ & $\begin{array}{l}\text { Tasa de crecimiento de los } \\
\text { impuestos indirectos. La } \\
\text { serie de define como el re- } \\
\text { sultado del cociente entre la } \\
\text { diferencia de los impuestos } \\
\text { indirectos pagados por los } \\
\text { hogares y los subsidios } \\
\text { entregados por el Estado a } \\
\text { las familias, y el gasto total } \\
\text { de estas. }\end{array}$ & $\begin{array}{l}\text { El efecto que se espera ante la } \\
\text { variación de los impuestos es, } \\
\text { en principio, ambiguo. Cuando } \\
\text { suben los impuestos indirec- } \\
\text { tos se reduce la capacidad } \\
\text { de consumo familiar, lo que } \\
\text { afecta al mercado de trabajo } \\
\text { de dos maneras. Por un lado, } \\
\text { los trabajadores en activo, para } \\
\text { mantener el poder adquisitivo, }\end{array}$ & (5) \\
\hline
\end{tabular}




\begin{tabular}{|c|c|c|c|}
\hline Variable & Concepto & $\begin{array}{c}\text { Efecto esperado en la tasa de } \\
\text { desempleo }\end{array}$ & Serie \\
\hline & & $\begin{array}{l}\text { reclaman incrementos } \\
\text { salariales que reducen las } \\
\text { posibilidades de coloca- } \\
\text { ción de los trabajadores } \\
\text { desempleados y aumentan } \\
\text { el desempleo. Por otro, los } \\
\text { trabajadores en desempleo } \\
\text { se ven obligados a reducir su } \\
\text { salario de reserva y a aceptar } \\
\text { puestos de trabajo a los que } \\
\text { antes renunciaban dada su } \\
\text { baja calidad o por no ser } \\
\text { acordes a su formación. } \\
\text { Dependiendo de cuál de los } \\
\text { dos efectos sea más intenso } \\
\text { el signo será positivo (primer } \\
\text { caso) o negativo (segundo } \\
\text { caso). }\end{array}$ & \\
\hline \multicolumn{4}{|c|}{$\begin{array}{l}\text { 1) La serie se ha tomado de Layard, Nickell y Jackman (1991) y se ha actualizado con la tasa } \\
\text { de desempleo ofrecida por la OECD, excepto en los casos de: Portugal, que se ha tomado } \\
\text { de la base de datos de la London School of Economics CEP-OECD hasta } 2004 \text { (Nickell } \\
\text { (2006) y Nickell y Nunziatta (2001)) y se ha actualizado con Ameco de la Comisión Europea; } \\
\text { Italia, cuyos datos se han recogido del United States Department of Labor; y Grecia, cuya } \\
\text { serie ha sido construida íntegramente con la base de datos Ameco. }\end{array}$} \\
\hline \multicolumn{4}{|c|}{$\begin{array}{l}\text { 2) La serie del índice de precios al consumo se ha obtenido de la base International Financial } \\
\text { Statistics (IFS) del Fondo Monetario Internacional. }\end{array}$} \\
\hline \multicolumn{4}{|c|}{$\begin{array}{l}\text { 3) La serie de productividad se ha construido como el cociente entre el PIB a precios } \\
\text { constantes y el empleo total. La serie del PIB real procede de las Cuentas Nacionales } \\
\text { publicadas por la OECD, mientras que la serie de empleo ha sido tomada de la base de } \\
\text { datos Economic Outlook de la OECD. }\end{array}$} \\
\hline \multicolumn{4}{|c|}{ 4) La serie de PIB real se ha tomado de las Cuentas Nacionales publicadas por la OECD. } \\
\hline \multicolumn{4}{|c|}{$\begin{array}{l}\text { 5) La serie de impuestos indirectos se ha tomado, entre } 1985 \text { y } 2004 \text {, de la base de datos } \\
\text { CEP-OECD de la London School of Economics (Nickell (2006)), y ha sido actualizada usando } \\
\text { las series de impuestos indirectos, subsidios totales y gasto privado total que ofrecen las } \\
\text { Cuentas Nacionales de la OECD. La excepción es Grecia, que se ha construido a partir de } \\
\text { las Cuentas Nacionales de la OECD y Ameco de la Comisión Europea. }\end{array}$} \\
\hline
\end{tabular}

Fuente: elaboración propia 
Efectos de las variables macroeconómicas e institucionales en el desempleo: análisis para Europa (1985-2011)

Tabla 4. Variables institucionales del mercado de trabajo y de productos consideradas en el estudio

\begin{tabular}{|c|c|c|c|}
\hline Variable & Concepto & Efecto esperado en la tasa de desempleo & Serie \\
\hline $\mathrm{COORD}_{i t}$ & $\begin{array}{l}\text { Variable que recoge el nivel } \\
\text { de coordinación que prevale- } \\
\text { ce en el régimen de negocia- } \\
\text { ción salarial, y en concreto el } \\
\text { grado de simultaneidad con } \\
\text { el que se llevan a cabo las } \\
\text { negociaciones en los dife- } \\
\text { rentes sectores o empresas. } \\
\text { El índice toma valores entre } 1 \\
\text { y } 5 \text {, tal que el valor } 5 \text { indica el } \\
\text { mayor grado de coordinación } \\
\text { posible. }\end{array}$ & $\begin{array}{l}\text { El efecto es ambiguo, pues una elevada coordi- } \\
\text { nación, al igual que una mínima coordinación, } \\
\text { mejora los resultados económicos y reducen la } \\
\text { tasa de desempleo. En negociaciones secto- } \\
\text { riales ocurre lo contrario. Blanchard, Joumotte } \\
\text { y Loungani (2013) sostienen que los niveles } \\
\text { intermedios de negociación incrementan el } \\
\text { poder negociador de los sindicatos, lo que } \\
\text { tiene efectos adversos sobre el empleo. }\end{array}$ & (1) \\
\hline $\operatorname{Min}_{i t}$ & $\begin{array}{l}\text { Variable que mide la relación } \\
\text { entre el salario mínimo y el } \\
\text { salario medio de la econo- } \\
\text { mía. Esta variable toma el } \\
\text { valor } 0 \text { para aquellos países } \\
\text { que como Austria, Dinamar- } \\
\text { ca, Finlandia, Alemania, Italia } \\
\text { y Suecia no tienen salario } \\
\text { mínimo interprofesional de } \\
\text { ámbito nacional durante el } \\
\text { período considerado en el } \\
\text { estudio } 6 \text {. }\end{array}$ & $\begin{array}{l}\text { Es de esperar un signo positivo. Cuanto más } \\
\text { alto sea el salario mínimo mayor será la tasa } \\
\text { de desempleo. Sin embargo, Card y Krueger } \\
\text { (1995) sostienen que no hay un consenso } \\
\text { claro sobre el impacto del salario mínimo en } \\
\text { la tasa de desempleo, pues éste afecta sólo } \\
\text { a trabajadores jóvenes con baja formación } \\
\text { y experiencia. El volumen de población que } \\
\text { cumple estas características es muy reducido, } \\
\text { sobre todo si se tiene en cuenta que como } \\
\text { mantienen Dolado et al. (1996) el efecto solo } \\
\text { es considerable en países en los que además } \\
\text { las cotizaciones sociales sean elevadas. }\end{array}$ & (2) \\
\hline $\mathrm{EPL}_{i t}$ & $\begin{array}{l}\text { Índice sintético que mide la } \\
\text { legislación a la contratación } \\
\text { y el despido de los trabajado- } \\
\text { res, y que representa el nivel } \\
\text { de protección que se ofrece } \\
\text { a los trabajadores. Su valor } \\
\text { se sitúa entre } 0 \text { y 5, siendo } \\
\text { las economías de Portugal, } \\
\text { Países Bajos, España, Grecia } \\
\text { e Italia, las que presentan un } \\
\text { mayor nivel de protección; } \\
\text { mientras que Reino Unido, } \\
\text { es el mercado más flexible. }\end{array}$ & $\begin{array}{l}\text { Es de esperar un signo positivo, pues la } \\
\text { legislación de protección a los empleados } \\
\text { reducen las posibilidades de las personas } \\
\text { desempleadas para abandonar su situación. El } \\
\text { motivo se debe a que las empresas tienden a } \\
\text { ser más cautelosas con las contrataciones. No } \\
\text { obstante, a corto plazo también reducen las } \\
\text { posibilidades de despido (Blanchard, Joumotte } \\
\text { y Loungani 2013)). } \\
\text { Blanchard y Giavazzi (2003) sos- } \\
\text { tienen que la desregulación del merca- } \\
\text { do de trabajo perjudica a corto plazo a } \\
\text { los trabajadores que tienen la seguridad }\end{array}$ & (3) \\
\hline
\end{tabular}

${ }_{6}$ Algunos países que no tienen salario mínimo interprofesional en el ámbito nacional, y para los que se ha considerado valor cero, tienen, sin embargo, un salario mínimo establecido para los trabajadores de algunos sectores. 


\begin{tabular}{|c|c|c|c|}
\hline Variable & Concepto & Efecto esperado en la tasa de desempleo & Serie \\
\hline & & $\begin{array}{l}\text { de ser contratados, ya que no genera sobre } \\
\text { ellos ningún efecto a largo plazo, salvo la } \\
\text { mayor probabilidad de ser despedidos, por } \\
\text { lo que la desregulación les resulta negativa. } \\
\text { Sin embargo, no ocurre lo mismo con los } \\
\text { trabajadores que tienen más probabilidades de } \\
\text { estar en desempleo, a los que la desregulación } \\
\text { les beneficia al aumentar sus posibilidades de } \\
\text { ser contratados. }\end{array}$ & \\
\hline TU & $\begin{array}{l}\text { Variable que mide el nivel de } \\
\text { sindicalización de la pobla- } \\
\text { ción trabajadora. Se calcula } \\
\text { como la proporción de traba- } \\
\text { jadores netos sindicalizados } \\
\text { frente al total de trabajadores } \\
\text { asalariados. }\end{array}$ & $\begin{array}{l}\text { Es de esperar un signo positivo, pues los } \\
\text { mayores niveles de sindicalización conllevan } \\
\text { un mayor poder negociador de los sindicatos, } \\
\text { y consecuentemente mayores niveles de } \\
\text { desempleo. }\end{array}$ & (4) \\
\hline Cot SS & $\begin{array}{l}\text { Variable que mide las co- } \\
\text { tizaciones al Sistema de } \\
\text { Seguridad Social a las que } \\
\text { hace frente el empresario, } \\
\text { expresadas como porcentaje } \\
\text { del PIB. }\end{array}$ & $\begin{array}{l}\text { Es de esperar un signo positivo, pues la coti- } \\
\text { zación a la seguridad social es un impuesto al } \\
\text { que debe hacer frente el empresario por cada } \\
\text { trabajador contratado. Cuanto mayor sea el } \\
\text { impuesto, mayor es el costo de contratar a } \\
\text { un trabajador y el desempleo aumentará. Los } \\
\text { trabajos de Nickell (1997), Scarpetta (1996), } \\
\text { Elmeskov, Martin y Scarpetta (1998), Daveri } \\
\text { y Tabellini (2000), Heitger (2002) y Melguizo y } \\
\text { González-Páramo (2013), entre otros, muestran } \\
\text { la existencia de esta relación positiva entre las } \\
\text { cargas tributarias sobre los empresarios y el } \\
\text { nivel de desempleo. }\end{array}$ & (5) \\
\hline APLM $_{i t}$ & $\begin{array}{l}\text { Variable que viene dada por } \\
\text { el cociente entre el gasto en } \\
\text { políticas activas del mercado } \\
\text { de trabajo y el gasto total } \\
\text { del gobierno en políticas } \\
\text { laborales }{ }^{7}\end{array}$ & $\begin{array}{l}\text { Las políticas activas tratan de favorecer la } \\
\text { colocación de los trabajadores, mientras } \\
\text { que las políticas pasivas buscan garantizar la } \\
\text { supervivencia de las personas desempleadas. } \\
\text { Por lo tanto, es de esperar que el signo sea } \\
\text { negativo. Un mayor gasto en políticas activas, } \\
\text { y siempre que éste se enfoque de manera } \\
\text { adecuada, debería incidir positivamente en } \\
\text { el mercado de trabajo con reducciones de la } \\
\text { tasa de desempleo. En sus trabajos empíricos, } \\
\text { Andersen y Svarer (2010) y Calmfors, }\end{array}$ & (6) \\
\hline
\end{tabular}

${ }_{7}$ Las políticas activas del mercado de trabajo incluyen las políticas de formación de la mano de obra, las ayudas en la búsqueda de empleo, los subsidios a la creación de empleo y las medidas especiales a favor de la colocación de los discapacitados. 
Efectos de las variables macroeconómicas e institucionales en el desempleo: análisis para Europa (1985-2011)

\begin{tabular}{|c|c|c|c|}
\hline \multirow[t]{2}{*}{ Variable } & Concepto & Efecto esperado en la tasa de desempleo & Serie \\
\hline & & $\begin{array}{l}\text { Forslund y Hemström (2004) encuentran en las } \\
\text { políticas activas una vía para la reducción del } \\
\text { desempleo. Por su parte Martin y Grubb (2001) } \\
\text { concluyen que su efecto no es significativo. }\end{array}$ & \\
\hline$U_{-} s c$ & $\begin{array}{l}\text { Variable que representa la } \\
\text { generosidad del seguro de } \\
\text { desempleo y, más en concre- } \\
\text { to, la tasa de sustitución del } \\
\text { seguro de desempleo. }\end{array}$ & $\begin{array}{l}\text { Es de esperar un signo positivo. En este senti- } \\
\text { do, Nickell (1997) sostiene que en la medida en } \\
\text { que el seguro de desempleo reduce el temor a } \\
\text { estar desempleado, aumenta la capacidad de } \\
\text { presión salarial de los trabajadores y dificulta } \\
\text { la colocación de las personas desempleadas. } \\
\text { Esta tesis es apoyada por Lalive y Zweimuller } \\
\text { (2004), Lalive, Ours y Zweimuller (2006), Ours } \\
\text { y Vodopivec (2006) y Adison y Portugal (2008). } \\
\text { No obstante, los trabajos de Ours y Vadopivec } \\
\text { (2008), Caliendo, Tatsiramos y Uhlendorff } \\
\text { (2009) y Barbanchon (2012), encuentran } \\
\text { un efecto positivo, aunque limitado de la } \\
\text { generosidad del seguro de desempleo en el } \\
\text { mercado de trabajo. }\end{array}$ & (7) \\
\hline $\mathrm{U}_{-} \mathrm{Dur}$, & $\begin{array}{l}\text { Indicador que mide la ge- } \\
\text { nerosidad del seguro de } \\
\text { desempleo al representar } \\
\text { la duración en semanas del } \\
\text { mismo. }\end{array}$ & $\begin{array}{l}\text { Es de esperar un signo positivo. La mayor } \\
\text { duración de los beneficios a los desempleados } \\
\text { se asocia con una tasa de desempleo más } \\
\text { elevada. El motivo reside en el hecho de que la } \\
\text { garantía de una renta desincentiva la búsqueda } \\
\text { activa de empleo. Esta afirmación ha sido } \\
\text { respaldada por los trabajos de Heitger (2002), } \\
\text { Farber y Valletta (2013) y Hagedor et al. (2013). }\end{array}$ & (7) \\
\hline 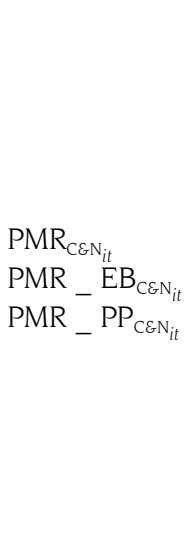 & $\begin{array}{l}\text { Indicadores agregados cons- } \\
\text { truido por Conway y Nicoletti } \\
\text { (2006) para los países de } \\
\text { la OECD, que miden, res- } \\
\text { pectivamente, la regulación } \\
\text { general en el mercado de } \\
\text { productos, las restricciones } \\
\text { existentes a la creación de } \\
\text { empresas, y la relevancia de } \\
\text { la propiedad pública y los } \\
\text { monopolios estatales. Sus } \\
\text { valores varían entre } 0 \text { y } 6 \text {, } \\
\text { siendo } 0 \text { el menor nivel de } \\
\text { regulación y } 6 \text { el mayor }{ }^{8} \text {. }\end{array}$ & $\begin{array}{l}\text { Es de esperar un signo negativo. Así, una } \\
\text { menor regulación en el mercado de productos, } \\
\text { la existencia de menores barreras a la creación } \\
\text { de empresas, o una menor intervención estatal } \\
\text { en el mercado, afectarán positivamente al } \\
\text { establecimiento de nuevos negocios y a la } \\
\text { creación de empleo. Los efectos, sin embargo, } \\
\text { son, como señalan Blanchard y Giavazzi } \\
\text { (2003), Jaumotte (2011) y Blanchard, Joumotte y } \\
\text { Loungani (2013), más a largo que a corto plazo. }\end{array}$ & (8) \\
\hline
\end{tabular}

$\overline{8}$ La serie se construye con información recogida de siete sectores no manufactureros (gas, electricidad, correos, telecomunicaciones y transporte aéreo, por ferrocarril o por carretera). Como los sectores no manufactureros representan casi dos tercios de la actividad económica, el indicador es una buena aproximación al nivel de regulación que impera en el mercado de productos en su conjunto. 


\begin{tabular}{|c|c|c|c|c|}
\hline \multicolumn{2}{|r|}{ Variable } & Concepto & Efecto esperado en la tasa de desempleo & Serie \\
\hline \multicolumn{2}{|c|}{ School_SCit } & $\begin{array}{l}\text { Os en } \\
\text { ación } \\
\text { lades } \\
\text { os } 25\end{array}$ & $\begin{array}{l}\text { A priori es de esperar un signo negativo, pues } \\
\text { cuanto mayor es la formación de la mano de } \\
\text { obra mayor es la posibilidad de encontrar } \\
\text { empleo. No obstante, la afirmación no puede } \\
\text { hacerse con carácter general, pues alcanzado } \\
\text { un determinado nivel de formación, y dada la } \\
\text { estructura productiva del país, podrían entrar } \\
\text { en funcionamiento los rendimientos margina- } \\
\text { les decrecientes, dificultando la contratación } \\
\text { de la mano de obra. Hecho que puede verse } \\
\text { agravado por la mayor productividad de la } \\
\text { mano de obra altamente cualificada }\end{array}$ & (9) \\
\hline \multicolumn{5}{|c|}{$\begin{array}{l}\text { 1) El índice de coordinación del mercado de trabajo se ha tomado, para el período 1985-2011, de } \\
\text { Visser (2013), cuyo índice se mueve en una escala que va de } 1 \text { a } 5 \text {. }\end{array}$} \\
\hline \multicolumn{5}{|c|}{$\begin{array}{l}\text { La serie del salario mínimo, así como la del salario medio, se ha tomado de la base de datos } \\
\text { Employment Outlook de la OECD. }\end{array}$} \\
\hline \multicolumn{5}{|c|}{$\begin{array}{l}\text { La serie de legislación sobre el mercado de trabajo está tomada de la OECD. En concreto se ha } \\
\text { optado por la serie de regulación de protección a favor del trabajador con contrato indefinido } \\
\text { que ofrece la base de datos Employment Outlook de la OECD. }\end{array}$} \\
\hline 4) & \multicolumn{4}{|c|}{$\begin{array}{l}\text { El nivel de densidad sindical se ha tomado, para el período 1985-2011, de Visser (2013). Su valor } \\
\text { varía entre } 0 \text { y } 100 .\end{array}$} \\
\hline 5) & \multicolumn{4}{|c|}{$\begin{array}{l}\text { Las cotizaciones de los empresarios a la Seguridad Social se han tomado de la serie Revenue } \\
\text { Statistics de la OECD. }\end{array}$} \\
\hline & \multicolumn{4}{|c|}{$\begin{array}{l}\text { Tanto la serie de gasto en políticas activas del mercado de trabajo como la del gasto total en } \\
\text { políticas laborales se han extraído del Programa de Políticas de Empleo que ofrece la base de } \\
\text { datos Employment Outlook de la OECD. }\end{array}$} \\
\hline & \multicolumn{4}{|c|}{$\begin{array}{l}\text { Las dos series que hacen referencia a la generosidad del seguro de desempleo (Unem_s_s, } \\
\text { Unem__ Dur) se han tomado de Scruggs, Jahn y Kuitto (2013) que ofrece valores para el período } \\
\text { 1970-2011. }\end{array}$} \\
\hline 8) & \multicolumn{4}{|c|}{$\begin{array}{l}\text { Conway y Nicoletti (2006) elaboran, para el período 1975-2007, un indicador sobre la regulación } \\
\text { del mercado de productos desde un punto de vista agregado y desagregado. En este último } \\
\text { caso cubre las barreras interpuestas por el gobierno a la creación de empresa, al comercio y la } \\
\text { inversión y a la propiedad pública. En concreto, se ha utilizado la serie de regulación agregada, } \\
\text { la que dificulta la creación de empresas, y la que muestra la intervención estatal en la economía. } \\
\text { Las series se han extendido hasta } 2011 \text { con las mismas series ofrecidas por la OECD y que han } \\
\text { sido elaboradas, siguiendo la misma dinámica, por los autores citados. }\end{array}$} \\
\hline \multicolumn{5}{|c|}{$\begin{array}{l}\text { La serie ha sido tomada de Cohen y Soto (2007), actualizada por Cohen y Leker (2014), quienes } \\
\text { facilitan datos decenales de formación de la mano de obra para el período 1960-2020. Para } \\
\text { disponer de datos anuales, se han interpolado linealmente los valores intermedios. }\end{array}$} \\
\hline
\end{tabular}

Fuente: elaboración propia 


\section{PRINCIPALES RESULTADOS}

Hechas las primeras valoraciones generales, corresponde ahora adentrarse en los resultados de las estimaciones realizadas. En concreto, se recurrió a un modelo MCO de datos de panel balanceado que se aplicó a los 14 países de la UE seleccionados?. En todos los casos, el número de observaciones por país es de 27, número adecuado para poder extraer efectos individuales ${ }^{10}$.

Para obtener las estimaciones se aplicó un proceso de iteración, de modo que las variables menos significativas se eliminaron hasta llegar a un modelo en el que todas las variables son relevantes con un nivel de significancia del $1 \%, 5 \%$ o $10 \%$, salvo en algún caso en el que se ha querido mostrar alguna variable cuyo nivel de significancia se aproxima mucho al $1 \%{ }^{11}$. Los resultados finales sobre las relaciones entre el desempleo y las variables macroeconómicas e institucionales del mercado de trabajo se presentan en la tabla 5.

\subsection{Variables macroeconómicas}

El estudio se inició con un primer modelo (modelo 1) que analiza el efecto de las variables macroeconómicas en el crecimiento del desempleo. De él se deduce que todas las variables consideradas, a excepción de la inflación, influyen, con mayor o menor intensidad, en la variación de la tasa de desempleo. Como cabía esperar, el crecimiento de la economía real guarda una relación estrecha y negativa con la variación del desempleo. Es, además, la variable con mayor impacto en el mercado de trabajo, seguida de la productividad de la mano de obra, cuyo incremento favorece la producción, pero reduce las necesidades de contratación afectando negativamente al empleo.

Asimismo, debe destacarse la incidencia negativa y significativa de la imposición indirecta, lo que significa que el aumento de los impuestos indirectos consigue reducir el desempleo. El aumento de los impuestos disminuye el poder adquisitivo de los ciudadanos, tanto de los trabajadores como de los desempleados, que se ven obligados a aceptar puestos de trabajo que antes rechazaban con el objetivo de mantener su poder de compra. El efecto ambiguo que en un principio habíamos previsto (tabla 3) se ha resuelto a favor de la reducción del desempleo. Así, durante el período analizado la subida de impuestos indirectos ha llevado a los desempleados a

\footnotetext{
Para corregir la estacionalidad de la tasa de desempleo, se ha aplicado el modelo a la tasa de crecimiento del desempleo.

10 Judson y Owen (1999) muestran el buen comportamiento de la estimación MCO en un panel suficientemente grande.

11 Las estimaciones se han realizado con el paquete econométrico Ox-Metrics 5.10.
} 
reducir su salario de reserva y aceptar puestos de trabajo a los que antes renunciaban. Este efecto que debemos considerar positivo para el mercado de trabajo tiene, sin embargo, su contrapartida negativa, pues los trabajadores desempleados que aceptan los puestos de trabajo vacantes para poder hacer frente a las necesidades básicas ante la subida de los impuestos, se ven obligados a aceptar puestos de trabajo de una calidad inferior a la que hubiesen deseado. En definitiva, baja el desempleo a costa de peores puestos de trabajo.

Tabla 5. Crecimiento del desempleo. Datos de panel de 14 países UE (MCO) (Variables macroeconómicas e institucionales del mercado de trabajo y productos) Datos anuales 1985-2011

\begin{tabular}{|c|c|c|c|c|c|}
\hline Variable & Modelo 1 & Modelo 2 & Modelo 3 & Modelo 4 & Modelo $5^{1}$ \\
\hline$d u_{t-1}$ & $\begin{array}{c}0,2374 \\
(3,63)^{* * *}\end{array}$ & $\begin{array}{c}0,2406 \\
(4,00)^{* * *}\end{array}$ & $\begin{array}{c}0,2428 \\
(4,02)^{* * *}\end{array}$ & $\begin{array}{c}0,2445 \\
(4,01)^{* * *}\end{array}$ & $\begin{array}{c}0,1689 \\
(1,760)^{* *}\end{array}$ \\
\hline Inf & & & & & $\begin{array}{c}-0,5485 \\
(-8,41)^{* * *}\end{array}$ \\
\hline $\mathrm{PIBg}$ & $\begin{array}{c}-4,6292 \\
(-9,71)^{* * *}\end{array}$ & $\begin{array}{c}-4,5451 \\
(-10,1)^{* * *}\end{array}$ & $\begin{array}{c}-4,6472 \\
(-11,0)^{* * *}\end{array}$ & $\begin{array}{c}-4,6157 \\
(-10,0)^{* * *}\end{array}$ & $\begin{array}{c}-4,7531 \\
(-14,8)^{* * *}\end{array}$ \\
\hline Prod & $\begin{array}{c}2,3195 \\
(2,85)^{* * *}\end{array}$ & $\begin{array}{c}2,2202 \\
(2,93)^{* * *}\end{array}$ & $\begin{array}{c}2,1999 \\
(3,01)^{* * *}\end{array}$ & $\begin{array}{c}2,1791 \\
(2,92)^{* * *}\end{array}$ & $\begin{array}{c}2,9080 \\
(7,04)^{* * *}\end{array}$ \\
\hline Itax & $\begin{array}{l}-0,1258 \\
(-1,39)^{*}\end{array}$ & $\begin{array}{l}-0,1423 \\
(-1,48)^{*}\end{array}$ & $\begin{array}{l}-0,1746 \\
(-1,89)^{* *}\end{array}$ & $\begin{array}{c}-0,1713 \\
(-1,80)^{* *}\end{array}$ & \\
\hline COORD & & $\begin{array}{c}0,0289 \\
(2,35)^{* * *}\end{array}$ & $\begin{array}{c}0,0307 \\
(2,62)^{* * *}\end{array}$ & $\begin{array}{c}0,0307 \\
(2,58)^{* * *}\end{array}$ & $\begin{array}{l}0,0272 \\
(1,47)^{*}\end{array}$ \\
\hline MinW & & $\begin{array}{c}0,0477 \\
(2,60)^{* * *}\end{array}$ & $\begin{array}{c}0,0834 \\
(3,10)^{* * *}\end{array}$ & $\begin{array}{c}0,0786 \\
(2,78)^{* * *}\end{array}$ & $\begin{array}{c}0,0812 \\
(2,37)^{* * *}\end{array}$ \\
\hline EPL & & $\begin{array}{c}0,0637 \\
(3,83)^{* * *}\end{array}$ & $\begin{array}{c}0,0351 \\
(2,21)^{* * *}\end{array}$ & $\begin{array}{l}0,0251 \\
(1,28)^{*}\end{array}$ & $\begin{array}{c}0,0331 \\
(2,13)^{* * *}\end{array}$ \\
\hline TU & & & & & \\
\hline Cot SS & & $\begin{array}{c}0,0246 \\
(2,57)^{* * *}\end{array}$ & $\begin{array}{c}0,0201 \\
(2,34)^{* * *}\end{array}$ & $\begin{array}{c}0,0241 \\
(2,50)^{* * *}\end{array}$ & \\
\hline APLM & & & & & $\frac{0,1030}{(1,86)^{* *}}$ \\
\hline
\end{tabular}


Efectos de las variables macroeconómicas e institucionales en el desempleo: análisis para Europa (1985-2011)

\begin{tabular}{|c|c|c|c|c|c|}
\hline Variable & Modelo 1 & Modelo 2 & Modelo 3 & Modelo 4 & Modelo $5^{1}$ \\
\hline$U_{-} s c$ & & $\begin{array}{c}-0,2022 \\
(-2,44)^{* * *}\end{array}$ & $\begin{array}{c}-0,16 \\
(-2,36)^{* * *}\end{array}$ & $\begin{array}{l}-0,1389 \\
(-1,98)^{* *}\end{array}$ & \\
\hline$U_{-} \mathrm{D} u r$ & & & & & $\begin{array}{c}0,0002 \\
(5,55)^{* * *}\end{array}$ \\
\hline $\mathrm{PMR}_{\mathrm{CEN}}$ & & & $\begin{array}{c}0,0116 \\
(3,66)^{* * *}\end{array}$ & & $\begin{array}{c}0,0109 \\
(3,85)^{* * *}\end{array}$ \\
\hline School_SC & & & & $\begin{array}{c}-0,0223 \\
(-2,46)^{* * *}\end{array}$ & \\
\hline & $R^{2}=0,644$ & $R^{2}=0,669$ & $\mathrm{R}^{2}=0,677$ & $R^{2}=0,675$ & $\mathrm{R}^{2}=0,677$ \\
\hline & No obs. 364 & No obs. 364 & $N^{\circ}$ obs. 364 & No obs. 364 & No obs. 208 \\
\hline & $\mathrm{N}^{\circ}$ var. 18 & $\mathrm{~N}^{\circ}$ var. 23 & $\mathrm{~N}^{\mathrm{O}}$ var. 24 & $\mathrm{~N}^{\mathrm{O}}$ var. 24 & $\mathrm{~N}^{\circ}$ var. 18 \\
\hline
\end{tabular}

NOTAS: 1 El modelo 5 testea solo los países que tienen salario mínimo interprofesional (Bélgica, Francia, Grecia, Irlanda, Países Bajos, Portugal, España y Reino Unido).

2 El número de variables incluye los efectos individuales de los países.

Variables significativas al $10 \%(*), 5 \%(* *)$ y $1 \%(* * *)$

Fuente: elaboración propia

\subsection{Variables macroeconómicas e institucionales del mercado de trabajo}

La incorporación de las variables institucionales del mercado de trabajo como variables explicativas del crecimiento del desempleo hace ganar significatividad a las variables macroeconómicas.

En cuanto a la significatividad de las primeras se debe indicar que, como se pone de manifiesto en el modelo 2 en el que se eliminan poco a poco todas las variables no significativas, al menos al nivel del $1 \%$, el salario mínimo interprofesional, las cotizaciones empresariales a la seguridad social, la regulación del mercado de trabajo y el grado de centralización en las negociaciones salariales favorecen el crecimiento del desempleo. Su signo positivo resulta coherente con las predicciones teóricas. Así, cuanto más caro le resulte al empresario contratar a un trabajador (salario mínimo y cotizaciones empresariales) o más difícil le resulte despedirle (regulación laboral), menor será su disposición a contratar y mayor el nivel de desempleo. En cuanto al signo positivo del grado de coordinación, resulta contrario a lo que en principio podría esperarse. La negociación salarial empresarial, existente en los países anglosajones, justificaría un signo positivo, pero la de ámbito nacional de los países nórdicos, justificaría un signo negativo. El signo positivo se explica por el mayor índice de coordinación que presentan los países con coordinación media, 
frente a los países anglosajones, que llevan a cabo negociaciones sectoriales y presentan elevadas tasas de desempleo.

Por su parte, el signo negativo de la generosidad del seguro de desempleo, medida a través de su tasa de reemplazo, pone de manifiesto que un seguro de desempleo mayor desincentiva la búsqueda activa de empleo. Siempre que el seguro de desempleo sea lo suficientemente generoso, las personas desempleadas no tendrán interés por incorporarse al mercado laboral, sobre todo si el salario que percibirían por trabajar es escasamente superior a la cantidad de dinero que se percibe del seguro de desempleo. Es más, algunos trabajadores que sienten la necesidad de incrementar sus ingresos tienden a acudir a la economía informal, y así realizan algún trabajo temporal o por horas, sin darse de alta en la seguridad social y sin tributar. De este modo pueden seguir cobrando el seguro de desempleo y complementarlo con los ingresos del trabajo realizado, de modo que la totalidad del dinero percibido suele superar a lo que obtendrían aceptando un puesto de trabajo. Esta situación suele ser habitual en los países de la cuenca mediterránea que, como España, Italia y Grecia, presentan tasas de desempleo más altas.

\subsection{Variables macroeconómicas e institucionales, tanto del mercado de trabajo como de productos, y formación de la mano de obra}

Los modelos 3 y 5 resultan de incorporar al análisis la regulación del mercado de productos. Mientras el primero considera la muestra total de países, el segundo elimina de la muestra los países que carecen de salario mínimo interprofesional. Por su parte, el modelo 4 incorpora las variables del modelo 3 y la formación de la mano de obra.

Al emplear el indicador global de regulación en el mercado de producto de Conway y Nicoletti (2006) en la estimación del modelo 3, se concluye que la intervención gubernamental en el mercado de productos es significativa y positiva, lo que implica que un aumento en la regulación acelerará el crecimiento del desempleo. El resto de variables que aparecen en el modelo, tanto las macroeconómicas como las institucionales del mercado de trabajo, son las mismas que aparecían en el modelo 2, y lo hacen con el mismo signo.

Si en lugar de medir el efecto de la regulación en el mercado de bienes y servicios, se incorpora al análisis la formación de la mano de obra (modelo 4), los resultados no cambian en exceso. La formación de la mano de obra resulta ser significativa en la determinación del desempleo y su signo negativo, lo que nos indica que un país con una población más formada tiene menos posibilidades de sufrir altas tasas de 
desempleo. En este sentido, aumentar los años medios dedicados a formación, tanto regulada como no regulada, aumenta las posibilidades de encontrar un empleo y también la productividad de la mano de obra, reduciendo consecuentemente el crecimiento del desempleo ${ }^{12}$. En cuanto al resto de variables, solo la regulación del mercado de trabajo y la generosidad del seguro de desempleo pierden algo de significatividad.

Por otro lado, la incorporación simultánea de la regulación en el mercado de productos y de la formación de la mano de obra expulsa a esta última variable, y deja el modelo 4 como modelo a considerar.

Hemos incorporado un quinto modelo (modelo 5), en el que hemos eliminado de la muestra los países que carecen de salario mínimo interprofesional. El objetivo es conocer si suponer que el salario mínimo toma valor 0 en los países que carecen del mismo, podría estar condicionando los resultados. Frente al modelo 3, que sí incorpora a los países sin salario mínimo, observamos que en las variables macroeconómicas desaparece como variable significativa la variación de los impuestos indirectos, mientras que aparece como variable que influye en la variación del desempleo los cambios en la tasa de inflación. El signo negativo con el que aparece la inflación permite a los gobiernos aplicar políticas expansivas que crearán inflación, pero que a cambio, al menos a corto plazo, podrán llegar a reducir el desempleo. Este signo negativo satisface la relación desempleo e inflación puesta de manifiesto en la curva de Phillips a corto plazo.

En cuanto a las variables institucionales del mercado de trabajo, tanto el salario mínimo interprofesional como la regulación del mercado de trabajo aparecen como variables explicativas del desempleo, y con el signo esperado. Cuanto mayor es el valor de ambas variables, mayor tiende a ser el desempleo en el país. Por otro lado, y frente al modelo 3 que considera a todos los países, aparece, en primer lugar, y sustituyendo a la tasa de sustitución del seguro de desempleo, la duración del mismo, también con el signo esperado, de modo que a mayor duración, y por lo tanto generosidad del seguro de desempleo, mayor es el desempleo existente, y en segundo lugar, el gasto en políticas activas del mercado de trabajo. No obstante, esta variable, a diferencia de lo que establece la teoría económica, tiene una incidencia positiva en el desempleo de los países considerados. Así, cuando mayor es el gasto en políticas activas, mayor es el desempleo. La explicación de este signo se encuentra, por un lado, en el gasto poco eficiente que los países analizados han hecho, en especial los países de la cuenca mediterránea (España, Grecia y Portugal), y por otro, en la escasa capacidad de las políticas activas para compensar los efectos que la crisis

12 Es la población con mayor formación, formación universitaria, la que ha mantenido su puesto de trabajo en los momentos de crisis, cuando el desempleo ha aumentado en todos los países. 
ha tenido sobre el empleo de dichos países. La coordinación en la negociación salarial se mantiene como variable explicativa del desempleo, si bien pierde parte importante de su significatividad.

Finalmente, una regulación elevada en el mercado de productos, y como ya ocurría en el modelo 3, explica también parte del desempleo existente.

\subsection{Test de robustez}

Una vez realizadas las estimaciones base, se han aplicado algunos test de robustez para determinar la validez del modelo. Los diferentes test se recogen en la tabla 6. Las dos primeras columnas comprueban la validez explicativa de la regulación del mercado de trabajo para lo que se utilizan dos indicadores diferentes. Así, se ha recurrido, en primer lugar al indicador construido por Nickell (2006) para el CEP-OECD de la London School of Economics, y en segundo, al índice de libertad regulatoria en el mercado de trabajo del Instituto Fraser ${ }^{13}$. Ambos indicadores resultan significativos, y como cabía esperar, el signo del primero es positivo, mientras el del segundo es negativo. Debe señalarse que ambos modelos se han estimado sin tener en cuenta la regulación en el mercado de productos.

Tabla 6. Test de robustez del análisis del crecimiento del desempleo. Datos de panel de 14 países UE (MCO)

\begin{tabular}{|c|c|c|c|c|c|c|c|c|}
\hline & $\begin{array}{c}\text { EPL } \\
\text { (Nickell) }\end{array}$ & EPL (Fraser) & Coord BO & CoordOchel & $\begin{array}{c}\text { Propiedad } \\
\text { pública }\end{array}$ & $\begin{array}{c}\text { Barreras de } \\
\text { entrada }\end{array}$ & $\begin{array}{c}\text { Modelo } 6 \\
\text { (1985-2006) }\end{array}$ & $\begin{array}{c}\text { Modelo } 7 \\
(1990-2011)\end{array}$ \\
\hline \multirow{2}{*}{ dut-1 } & 0,2304 & 0,2383 & 0,2452 & 0,2465 & 0,2435 & 0,2408 & 0,2928 & 0,2053 \\
\hline & $(3,96)^{* * *}$ & $(3,83)^{* * *}$ & $(3,89)^{* * *}$ & $(3,95)^{* * *}$ & $(4,00)^{* * *}$ & $(3,99)^{* * *}$ & $(6,36)^{* * *}$ & $(3,39)^{* * *}$ \\
\hline \multicolumn{9}{|l|}{ Inf } \\
\hline \multirow{2}{*}{$\mathrm{PIBg}$} & $-4,5426$ & $-4,5815$ & $-4,6824$ & $-4,666$ & $-4,6466$ & $-4,6333$ & $-4,8516$ & $-4,7498$ \\
\hline & $(-9,60)^{* * *}$ & $(-10,0)^{* * *}$ & $(-11,2)^{* * *}$ & $(-11,2)^{* * *}$ & $(-10,8)^{* * *}$ & $(-11,0)^{* * *}$ & $(-7,54)^{* * *}$ & $(-11,4)^{* * *}$ \\
\hline \multirow{2}{*}{ Prod } & 2,2526 & 2,3065 & 2,1512 & 2,1375 & 2,2019 & 2,1971 & 1,6128 & 2,041 \\
\hline & $(2,95)^{* * *}$ & $(2,81)^{* * *}$ & $(2,94)^{* * *}$ & $(2,95)^{* * *}$ & $(2,99)^{* * *}$ & $(3,00)^{* * *}$ & $(2,15)^{* * *}$ & $(2,64)^{* * *}$ \\
\hline \multirow{2}{*}{ Itax } & $-0,1516$ & $-0,11397$ & $-0,1397$ & $-0,146$ & $-0,1736$ & $-0,1663$ & & \\
\hline & $(-1,66)^{* *}$ & $(-1,61)^{*}$ & $(-1,84)^{* *}$ & $(-1,92)^{* *}$ & $(-1,90)^{* *}$ & $(-1,77)^{* *}$ & & \\
\hline \multirow{2}{*}{ COORD } & 0,0286 & 0,0251 & 0,038 & 0,03007 & 0,0302 & 0,0305 & 0,0343 & 0,0304 \\
\hline & $(2,41)^{* * *}$ & $(2,04)^{* * *}$ & $(1,70)^{* *}$ & $-1,26$ & $(2,60)^{* * *}$ & $(2,57)^{* * *}$ & $(2,47)^{* * *}$ & $(2,05)^{* * *}$ \\
\hline
\end{tabular}

13 El índice EPL de Nickell (2006) se ha extendido con la serie de regulación protectora a favor del trabajador que para los trabajadores con contrato indefinido ofrece la base de datos Employment Outlook de la OECD (2016a). En el caso de Grecia, para la que Nickell no ofrece datos, se ha utilizado la serie re-escalada que ofrece esta última base de datos. 
Efectos de las variables macroeconómicas e institucionales en el desempleo: análisis para Europa (1985-2011)

\begin{tabular}{|c|c|c|c|c|c|c|c|c|}
\hline & $\begin{array}{c}\text { EPL } \\
\text { (Nickell) }\end{array}$ & EPL (Fraser) & Coord BO & CoordOchel & $\begin{array}{l}\text { Propiedad } \\
\text { pública }\end{array}$ & $\begin{array}{c}\text { Barreras de } \\
\text { entrada }\end{array}$ & $\begin{array}{c}\text { Modelo } 6 \\
(1985-2006)\end{array}$ & $\begin{array}{c}\text { Modelo } 7 \\
(1990-2011)\end{array}$ \\
\hline MinW & $\begin{array}{c}0,0856 \\
(2,16)^{* * *}\end{array}$ & $\begin{array}{c}0,0644 \\
(3,08)^{* * *}\end{array}$ & $\begin{array}{c}0,0876 \\
(2,97)^{* * *}\end{array}$ & $\begin{array}{c}0,0909 \\
(3,29)^{* * *}\end{array}$ & $\begin{array}{c}0,0801 \\
(2,71)^{* * *}\end{array}$ & $\begin{array}{c}0,0734 \\
(3,03)^{* * *}\end{array}$ & $\begin{array}{c}0,1107 \\
(3,19)^{* * *}\end{array}$ & $\begin{array}{c}0,1163 \\
(4,73)^{* * *}\end{array}$ \\
\hline EPL & $\begin{array}{c}0,0737 \\
(2,21)^{* * *}\end{array}$ & $\begin{array}{c}-0,0064 \\
(-1,31)^{*}\end{array}$ & $\begin{array}{l}0,0242 \\
(1,61)^{*}\end{array}$ & $\begin{array}{c}0,0244 \\
(1,51)^{*}\end{array}$ & $\begin{array}{c}0,0412 \\
(2,71)^{* * *}\end{array}$ & $\begin{array}{c}0,0354 \\
(2,06)^{* * *}\end{array}$ & $\begin{array}{l}0,0302 \\
(1,36)^{*}\end{array}$ & \\
\hline \multicolumn{9}{|l|}{ TU } \\
\hline Cot SS & $\begin{array}{c}0,0207 \\
(2,21)^{* * *}\end{array}$ & $\begin{array}{c}0,0212 \\
(2,38)^{* * *}\end{array}$ & $\begin{array}{c}0,0204 \\
(2,25)^{* * *}\end{array}$ & $\begin{array}{c}0,0208 \\
(2,69)^{* * *}\end{array}$ & $\begin{array}{c}0,0205 \\
(2,38)^{* * *}\end{array}$ & $\begin{array}{c}0,0208 \\
(2,40)^{* * *}\end{array}$ & $\begin{array}{c}0,0177 \\
(1,67)^{* *}\end{array}$ & $\begin{array}{c}0,0293 \\
(3,74)^{* * *}\end{array}$ \\
\hline APLM & $\begin{array}{l}0,0811 \\
(-1,13)\end{array}$ & & & & & & & \\
\hline $\mathrm{U}_{-} s \mathrm{sc}$ & $\begin{array}{l}-0,1602 \\
(-1,87)^{* *}\end{array}$ & $\begin{array}{l}-0,1532 \\
(-1,58)^{*}\end{array}$ & $\begin{array}{c}-0,1844 \\
(-2,51)^{* * *}\end{array}$ & $\begin{array}{c}-0,2094 \\
(-2,41)^{* * *}\end{array}$ & $\begin{array}{c}-0,1703 \\
(-2,58)^{* * *}\end{array}$ & $\begin{array}{c}-0,1655 \\
(-2,41)^{* * *}\end{array}$ & & \\
\hline \multicolumn{9}{|l|}{$\mathrm{U}_{-} \mathrm{D} u r$} \\
\hline $\mathrm{PMR}_{\mathrm{CEN}}$ & & & $\begin{array}{c}0,0115 \\
(3,89)^{* * *}\end{array}$ & $\begin{array}{c}0,0114 \\
(3,68)^{* * *}\end{array}$ & $\begin{array}{c}0,0167 \\
(3,54)^{* * *}\end{array}$ & $\begin{array}{c}0,0086 \\
(3,30)^{* * *}\end{array}$ & $\begin{array}{r}0,005 \\
-1,07\end{array}$ & $\begin{array}{c}0,0224 \\
(4,48)^{* * *}\end{array}$ \\
\hline \multicolumn{9}{|c|}{ School_SC } \\
\hline & $\mathrm{R}^{2}=0,670$ & $R^{2}=0,665$ & $R^{2}=0,666$ & $R^{2}=0,666$ & $\mathrm{R}^{2}=0,677$ & $R^{2}=0,676$ & $\mathrm{R}^{2}=0,661$ & $\mathrm{R}^{2}=0,706$ \\
\hline & $\mathrm{n}^{\circ}$ ob. 364 & $\mathrm{n}^{\circ}$ ob. 364 & $\mathrm{n}^{\circ}$ ob. 364 & $\mathrm{n}^{\circ}$ ob. 364 & no ob. 364 & $\mathrm{n}^{\circ}$ ob. 364 & no ob. 294 & $\mathrm{n}^{\circ}$ ob. 294 \\
\hline & $\mathrm{n}^{\circ}$ var. 24 & $\mathrm{n}^{\circ}$ var. 23 & $\mathrm{n}^{\circ}$ var. 24 & $\mathrm{n}^{\circ}$ var. 24 & $\mathrm{n}^{\circ}$ var. 24 & $\mathrm{n}^{\circ}$ var. 24 & $\mathrm{n}^{\circ}$ var. 22 & $\mathrm{n}^{\circ}$ var. 21 \\
\hline
\end{tabular}

Notas: 1 El número de variables incluye los efectos individuales de los países.

Variables significativas al $10 \%\left({ }^{*}\right), 5 \%(* *)$ y $1 \%(* * *)$

\section{Fuente: elaboración propia}

Las dos siguientes columnas testean el grado de centralización en la negociación salarial para lo que utilizan los indicadores construidos por Belot y Ours (2001) y DICE $^{14}$. Mientras en el primer caso la capacidad explicativa de la negociación salarial se mantiene, aunque pierde intensidad, en el segundo la variable no resulta suficientemente significativa. En la quinta y sexta columnas de la tabla 6 se testea la validez de la regulación del mercado de productos, para lo que se hace uso de dos indicadores parciales de Conway y Nicoletti (2006), en concreto, la propiedad o control público en el mercado, y las barreras a la creación de empresas. Ambos indicadores resultan significativos, y mantienen la intensidad explicativa y el signo del resto de variables.

Las dos últimas columnas testean, con las variables inicialmente consideradas, períodos de tiempo diferentes. El modelo 6 excluye del análisis el período de crisis

14 Database for Institutional Comparisons in Europe (DICE). Ambas series son ofrecidas por Nickell (2006), y se han extendido con los datos de coordinación del mercado de trabajo de Visser (2013). 
iniciado en 2007. En este caso, la regulación del mercado de productos pierde capacidad explicativa. Por su parte, en el modelo 7, que excluye del estudio los años iniciales (1985-1989), resta validez a la regulación del mercado de trabajo como variable explicativa del crecimiento del desempleo, que desaparece del modelo, al tiempo que aumenta la intensidad con la que el salario mínimo interprofesional, las cotizaciones empresariales a la seguridad social y la regulación del mercado de productos inciden en el crecimiento del desempleo. Estos hechos parecen poner de manifiesto que son estas las variables sobre las que se debe actuar para dar solución al problema del desempleo y para conseguir una mayor convergencia real, en concreto en términos de empleo, entre los países europeos.

\section{CONCLUSIONES}

Del análisis realizado se pueden extraer algunas conclusiones y recomendaciones de política económica que podrían ayudar, por un lado, a reducir el elevado nivel de desempleo que, de forma permanente, presentan algunos países europeos, y por otro, aumentar la convergencia real entre los países de la Unión Europea.

En relación con las principales conclusiones que pueden extraerse del análisis realizado cabe señalar, en primer lugar, que existen diferencias importantes en el comportamiento del desempleo entre unos países y otros. Estas diferencias no se deben solo al comportamiento cíclico de la economía, y consecuentemente a las variables macroeconómicas, sino también a factores estructurales que permanecen en el tiempo. Entre estos factores estructurales se destaca la excesiva regulación, tanto del mercado de trabajo como del mercado de productos, síntomas claros de la rigidez económica de algunos países.

En segundo lugar, y aunque las diferencias que los países presentan en los niveles de regulación de ambos mercados (trabajo y bienes) son importantes, no son suficientes para explicar las tasas de desempleo que muestran los países. Es necesario destacar la existencia de otras variables que acompañan a las dos primeras a la hora de explicar el funcionamiento diferente del mercado laboral, en concreto, el salario mínimo interprofesional y las cotizaciones empresariales a la seguridad social.

En tercer lugar, y si bien las variables institucionales justifican la permanencia del desempleo, son las macroeconómicas las que resultan esenciales para iniciar el proceso, así como para explicar el comportamiento cíclico del mercado laboral. La caída del crecimiento económico y el comportamiento de la productividad de la mano de obra son, en este sentido, variables relevantes. Si bien, la estructura productiva de ciertos países, y más en concreto los sectores intensivos en mano de obra de baja cualificación que predominan en algunos, impulsan, por un lado, 
el fuerte comportamiento cíclico del desempleo, y por otro, las mayores tasas de desempleo a largo plazo.

De estas conclusiones pueden extraerse algunas recomendaciones de política económica que, adecuadamente aplicadas, ayudarían a los países con mayores problemas laborales a acercar sus posiciones a las de los países con menores tasas de desempleo y lograr así una convergencia real, al menos en lo que al nivel de desempleo se refiere. Entre estas posibles medidas encontramos:

1. Para solucionar problemas de desempleo a corto plazo es posible actuar sobre los impuestos indirectos. No obstante, esta medida solo puede ser temporal. El motivo se debe a que el incremento de los impuestos reduce la capacidad de consumo de los ciudadanos y los impulsa a aceptar puestos de trabajo que en otras condiciones no aceptarían, bien por la poca estabilidad del empleo, el bajo sueldo o no ser un empleo acorde con la formación del trabajador. En definitiva, la subida de los impuestos indirectos puede llegar a reducir el desempleo, pero a costa de puestos de trabajo de baja calidad. El objetivo de cualquier gobierno debe ser crear empleo de calidad, que favorezca la estabilidad y el crecimiento económico en el medio y largo plazo. Las reducciones de los impuestos indirectos son, por tanto, una solución al desempleo, aunque no es la más óptima para los ciudadanos de un país.

2. Si el objetivo es a más largo plazo, es más adecuado actuar sobre el nivel o la estructura del salario mínimo interprofesional. Una reducción del mismo, o incluso el establecimiento de niveles salariales distintos para diferentes grupos de población puede ayudar a reducir el desempleo. Está demostrado que el salario mínimo interprofesional no genera desempleo en las personas con cierto nivel de formación y experiencia. Son las personas sin formación y normalmente muy jóvenes las que sufren las consecuencias de la existencia de los salarios mínimos interprofesionales. Establecer niveles salariales diferentes según el nivel de formación y la edad de las personas podría ayudar a reducir el desempleo.

3. Reducir las cotizaciones empresariales a la Seguridad Social, o crear unas condiciones favorables a la negociación salarial en el ámbito de empresa pueden ser medidas más efectivas para reducir el desempleo y apoyar la convergencia real entre los Estados miembros de la Unión Europea. En este sentido, y como señalan los recientes trabajos de Blanchard, Joumotte y Loungani (2013), Álvarez-Martínez y Polo (2014) y Kratena y Sommer (2014), una reducción de las cotizaciones a la seguridad social permitiría mejorar la competitividad y, por lo tanto, aumentar las exportaciones. Esto crearía un ambiente adecuado 
para la generación de empleo. Esta medida resulta adecuada para los países considerados, pues como mantienen Mooij y Keen (2012), los efectos positivos de esta medida requieren de un tipo de cambio fijo, así como de rigidez al alza del salario nominal (ambas condiciones se dan en el caso de la Unión Europea). Solo así logrará evitarse la apreciación de la moneda nacional y la recuperación de los costos de producción, que compensarían los efectos positivos de la medida. En cualquier caso, los efectos, como sostienen Koske (2013) y Bosca, Doménech y Ferri (2013), son reducidos, por lo que hay que esta medida debe acompañarse de medidas adicionales.

4. Desregular el mercado laboral puede tener efectos positivos sobre la contratación. En este sentido, reducir las indemnizaciones por despido a las que debe hacer frente el empresario aumenta las posibilidades de encontrar un empleo, pues genera seguridad en el empresario. Junto a ello, reducir la generosidad del seguro de desempleo e incluir ciertos controles, por parte de la Administración Pública, que reduzcan el fraude y eviten que los desempleados rechacen puestos de trabajo, o que, incluso, acepten hacer trabajos en la economía informal a cambio de poder seguir cobrando el seguro de desempleo incentiva la búsqueda activa de empleo ${ }^{15}$. Aunque ambas medidas de manera conjunta pueden ayudar a reducir el desempleo, lo cierto es que generan inseguridades en el trabajador que ve cómo se reducen las rentas que obtendría en caso de despido y se siente más desprotegido. Para evitar estas inseguridades es posible incorporar la denominada mochila austriaca que permite distribuir el costo de la indemnización por despido entre todos los años que el trabajador se encuentra en la empresa, y que, además, se traslada con el trabajador cuando éste cambia de empresa. De esta manera se favorece la movilidad de la mano de obra lo que flexibiliza el mercado laboral y reduce el desempleo. Hay que tener en cuenta que en muchos países la cuantía de la indemnización por despido depende de los años que el trabajador haya estado en la empresa, lo que hace que estos se sientan reacios a cambiar de empleo introduciendo rigidez en el mercado laboral.

5. Crear un marco jurídico y económico adecuado que incremente la competencia en el mercado de productos, reduciendo la intervención estatal y las barreras a la creación de empresas. Ambas medidas ayudarán a la creación de empresas aumentando los puestos de trabajo y reduciendo consecuentemente el desempleo.

15 El trabajo de Andrés y Doménech (2015) profundiza en el efecto que sobre el desempleo genera la ausencia de controles por parte de la Administración Pública cuando el seguro de desempleo es generoso. 
Efectos de las variables macroeconómicas e institucionales en el desempleo: análisis para Europa (1985-2011)

6. Todas estas medidas, acompañadas de incentivos al desarrollo de sectores de alta tecnología y de una formación de calidad de la mano de obra, ayudarán a crear empleo estable y productivo.

\section{BIBLIOGRAFÍA}

Adascalitei, D. y Pignatti, C. (2015). Labour market reforms since the crisis: Drivers and consequences. En: Working Paper, No 5, International Labour Office, Ginebra, 27p.

Addison, J. T. y Portugal, P. (2008). How to Different Entitlements to Unemployment Benefits Affect the Transitions from Unemployment into Employment? En: Economics Letters Vol. 101, No.3, p. 206-209.

Álvarez-Martínez, M.T. y Polo, C., (2014). Reduce Social Security Contributions and Control Labour Fraud: Spain's Ailing Economy. En: Economic Systems Research, Vol. 26, No 2, p. 141-153.

Andersen, T. y Svarer, M. (2010). Do Temporary Help Jobs Improve Labour Market outcomes for Low-Skilled Workers? Evidence from Work First. En: American Economic Journal: Applied Economics, Vol 2, No 3, p. 96-128.

Andrés, J. y Doménech, R. (2015). En busca de la prosperidad. Capítulo 2: Más y mejor empleos, Editorial Deusto, Madrid, España, noviembre, 384p.

Barbanchon, T. (2012). The Effect of the Potential Duration of Unemployment Benefits on Unemployment Exists to Work and Match Quality in France. En: Centre de Rechercheen Économieset Statistique Série des Documents de Travail No $\overline{201} 2-21$, France, 52p.

Belot, M. y van Ours, J. (2001). Unemployment and Labour Market Institutions; an Empirical Analysis. En: Journal of the Japanese and International Economies Vol. 15, No 4, p. 403-418.

Blanchard, O. y Giavazzi, F. (2003). Macroeconomic Effects of Regulation and Deregulation in Goods and Labor Markets. En: Quarterly Journal of Economics Vol. 118, No. 3, p. 879-907.

Blanchard, O.;Joumotte, F. y Loungani, P. (2013). Labor Market Policies and IMF Advice in Advanced Economies During the Great Recession. En: IMF Staff Discussion Note, SDN/13/02, 2013. 30p.

Boscá, J. E.; Doménech, R. y Ferri, J. (2013). Fiscal Devaluation in EMU. En: BBVA Research Working Paper, No 12/11, Madrid, 38p.

Cacciatore, M.; Duval, R.; Fiori, G. y Ghironi, F. (2016). Short-Term Pain for Long-Term Gain: Market Deregulation and Monetary Policy in Small Open Economies. En: Journal of International Money and Finance, Vol. 68, noviembre, p 358-385.

Caliendo, M.; Tatsiramos, K. y Uhlendorff, A. (2009).Benefit Duration, Unemployment Duration and Job Match Quality: A Regression-discontinuity Approach. En: IZA Discussion Paper No 4670, 37p.

Calmfors, L.; Forslund, A. y Hemström, M. (2004): The Effects of Active Labour Market Policies in Sweden: What is the Evidence? En: Agell, J., Keen, M. and Weichenrieder, A., Labour Market Institutions and Public Regulations, Ed. MIT Press, Cambridge, 62p. 
Card, D. y Krueger, A. (1995). Myth and Measurement: the New Economics of the Minimum Wage, Ed. Princeton University Press, New Jersey, 422p.

Cohen, D. y Leker, L. (2014).Health and Education: Another Look with the Proper Data. mimeo, 25p.

Cohen, D. y Soto, M. (2007).Growth and human capital: good data, good results. En: Journal of Economic Growth Vol. 12, No 1, p. 51-76.

Conway, P. y Nicoletti, G. (2006). Product Market Regulation in the Non- manufacturing Sectors of OECD Countries: Measurement and Highlights. En: Working Paper No 58, Economics Department OECD, 64p.

Daveri, F. y Tabellini, G. (2000).Unemployment, Growth and Taxation in Industrial Countries. En: Economic Policy Vol.15, No 30, p. 47-104.

Dolado, J.; Kramarz, F.; Machin, S.; Manning, A.; Margolis, B. y Teulings, C. (1996). The Economic Impact of Minimum Wages in Europe. En: Economic Policy Vol.11, No.23, p. 317-373.

Elmeskov, J.; Martin, J. P. Y Scarpetta, S. (1998). Key Lessons for Labour Market Reforms: Evidence from OECD Countries' Experiences. En: Swedish Economic Policy Review Vol. 5, No 2, p. 205-252.

Farber, H. S. y Valletta, R. G. (2013). Do Extended Unemployment Benefits Lengthen Unemployment Spells? Evidence from Recent Cycles in the U.S. Labor Market. En: Federal Reserve Bank of San Francisco Working Paper, No 2013-09, 43p.

Hagedor, M.; Karahan, F.; Manovskii, I. y Mitman, K. (2013). Unemployment benefits and unemployment in the Great Recession: The role of Macro effects. En: Federal Reserve Bank of New York Staff Report, No 646, 77p.

Heitger, B. (2002). The impact of Taxation on Unemployment in OECD Countries. En: Cato Journal, Vol. 22, No. 2, p. 333-354.

Jaumotte, F. (2011). The Spanish labor market in a cross-country perspective. En: IMF Working paper, No. 11/11, Washington, 52p.

Judson, R. A. y Owen, A. L. (1999). Estimating dynamic panel data models: a guide for macroeconomists, En: Economic Letters, Vol. 65, No 1, p. 9-15.

Koske, I. (2013). Fiscal Devaluation: Can it Help to Boost Competitiveness? En: OECD Economic Department Working Paper, No.1089, Paris, 16p.

Kratena, K. y Sommer, M. (2014). Labour market policy and environmental fiscal devaluation: a cure for Spain in the aftermath of the great recession? En: WIFO Working paper, No. 476/2014, Viena, 56p.

Lalive, R. y Zweimuller, J. (2004). Benefit Entitlement and Unemployment Duration: The Role of Policy Endogeneity. En: Journal of Public Economic Vol. 88, No 12, p. 2587-2616.

Lalive, R.; van Ours, J. y Zweimuller, J. (2006). How Changes in Financial Incentives Affect the Duration of Unemployment. En: Review of Economics Studies, Vol. 73, No 4, p. 1009-1038.

Layard, R.; Nickell, S. y Jackman, R. (1991). Unemployment: Macroeconomic Performance and the Labour Market, Ed. Oxford University Press, London, 623p. 
Efectos de las variables macroeconómicas e institucionales en el desempleo: análisis para Europa (1985-2011)

Martin, J. y Grubb, D. (2001). What Works and for Whom: a Review of OECD Countries' Experiences with Active Labour Market Policies, En: Swedish Economic Review, Vol. 8, No 2, p. 9-56.

Melguizo, A. y González-Páramo, J. M. (2013). Who bears labour taxes and social contributions. En: SERIEs Spanish Economic Association, Vol. 4, No. 3, p. 247-271.

Mooij, R. y Keen, M. (2012). Fiscal Devaluation and Fiscal Consolidation: The VAT in troubled times, En: IMF Working Paper No. 12/85, Washington, 48p.

Nickell, S. J. (2006). The CEP-OECD Institutions Dataset (1960-2004). CEP Discussion Paper No. 759, CEP-LSE, 22p.

Nickell, S. (1997). Unemployment and Labour Market Rigidities: Europe versus North America. En: Journal of Economic Perspectives Vol. 11, No. 3, p 55-74.

Nickell, S. J. y Nunziata, L. (2001). Labour Market Institutions Database, versión 2.00, 1960-1995, CEP-LSE.

Ochel, W., (2001). Collective bargaining coverage in the OECD from 1960,s to the 1990s. En: CESIfo Forum, Vol.2, No. 4, p 62-65.

Ours, J. y Vodopivec, M. (2008). Does Reducing Unemployment Insurance Generosity Reduce Job Match Quality? En: Journal of Public Economics, Vol. 92, No 3-4, p. 684-395.

Ours, J. y Vodopivec, M. (2006). How Shortening the Potential Duration of Unemployment Benefits Affects the Duration of Unemployment: Evidence from a Natural Experiment. En: Journal of Labor Economics, Vol. 24, No 2, p. 351-378.

Pampillón Olmedo, R. y Mingorance Arnáiz, A. C. (2014). La lucha contra el paro. Una perspectiva internacional, p. 43-66. En: Marín, M. (Dir) y Bote, V. (Coodir.). Reflexiones sobre el mercado de trabajo: continuar la reforma. Ed. Fundación para el análisis y los estudios sociales (FAES), Madrid, 228p.

Pérez, E. y Yao, Y. (2012). Can Institutional Reform Reduce Job Destruction and Unemployment Duration? Yes it Can. En: IMF Working Paper, No. 12/54, Washington, 25p.

Scarpetta, S. (1996). Assessing the Role of Labour Market Policies and Institutional Settings on Unemployment: a Cross-Country Study. En: OECD Economic Studies, No. 26, p. 43-98.

Scruggs, L.; Jahn, D. y Kuitto, K. (2013). Comparative Welfare Entitlements Dataset, versión 2013 08, base de datos. Disponible en web (http://cwed2.org/).

Visser, J. (2013). ICTWSS Database, Database on Institutional Characteristics of Trade Unions, Wage Setting, State Intervention and Social Pacts in 34 countries between 1960 and 2011, versión 4.00, Ed. Institute for Advanced Labour Studies, AIAS, University of Amsterdam, Amsterdam. 
